\title{
Which medicine? Whose standard? Critical reflections on medical integration in China
}

\author{
Ruiping Fan, lan Holliday
}

J Med Ethics 2007;33:454-461. doi: 10.1136/jme.2006.017483

There is a prevailing conviction that if traditional medicine (TRM) or complementary and alternative medicine (CAM) are integrated into healthcare systems, modern scientific medicine (MSM) should retain its principal status. This paper contends that this position is misguided in medical contexts where TRM is established and remains vibrant. By reflecting on the Chinese policy on three entrenched forms of TRM (Tibetan, Mongolian and Uighur medicines) in western regions of China, the paper challenges the ideology of science that lies behind the demand that all traditional forms of medicine be evaluated and reformed according to MSM standards. Tibetan medicine is used as a case study to indicate the falsity of a major premise of the scientific ideology. The conclusion is that the proper integrative system for TRM and MSM is a dual standard based system in which both TRM and MSM are free to operate according to their own medical standards.

See end of article for authors' affiliations

Correspondence to: Professor Ruiping Fan, Department of Public and Social Administration, City University of Hong Kong, Kowloon, Hong Kong; safan@cityu.edu.hk

Received 24 May 2006

Revised 9 August 2006

Accepted 16 August 2006
T raditional medicine (TRM) and complementary and alternative medicine (CAM) are attracting growing attention. However, the prevailing conviction is that if these forms of medicine are integrated into healthcare systems, modern scientific medicine (MSM) should retain its principal status. In this article, we contend that this position is misguided in medical contexts where TRM is established and remains vibrant. ${ }^{\mathrm{i}}$ In this article, attention is not directed to TRM as it has developed among the Han people of China. This form of TRM roughly constitutes what most in the West identify as "Chinese medicine"; and has been explored elsewhere. ${ }^{12}$ Against those who take the dominance of MSM for granted, we argue that in order to develop an appropriate integrative system for MSM and TRM it is first necessary to explore two fundamental questions. Which medicine should be emphasised? Whose medical standard should be adopted?

These fundamental questions have not been seriously addressed. The dominant position is implicit in World Health Organization (WHO) documents that promote the claims of TRM within an integrative system. WHO states that "[i]n an integrative system, TM/CAM is officially recognised and incorporated into all areas of healthcare provision". ${ }^{3}$ However, "officially recognised and incorporated" is a vague requirement, implying an open-ended spectrum. At one extreme, there is presumably considerable state support for research and education in TRM practices, and TRM thera- pies are widely available from hospitals and clinics. At the other extreme, TRM may have no more than a limited presence, even though it is indeed present in "all areas": research, education, clinical practice and pharmacy. In such a case, incorporation of TRM into a so-called "integrative system" is no more than token, which is clearly problematic.

The question of "whose standard" is even more fundamental. Every medicine embodies a medical standard in the form of a set of professionally and popularly approved norms, rules and mechanisms by which specific diagnostic, therapeutic and pharmaceutical practices (including products) are validated for use in healthcare settings. Evidently, there are as many different and incommensurable medical standards as there are various and incommensurable medical traditions. In order to set up an integrative system, such as for TRM and MSM, government therefore needs to formulate a policy regarding the medical standard that is to be adopted for selecting, operating and regulating integrative practice. In principle, there are several possibilities: TRM standard based, MSM standard based, dual standard based (whereby TRM and MSM operate independently according to their own standards), and new standard based (whereby a new standard is framed out of mixed TRM and MSM standards). Which of these possible standards should inform integration of TRM and MSM?

This paper explores these issues by reflecting on the special case of China. In particular, it focuses on three formally autonomous regions in the north and west: Inner Mongolia, Tibet and Xinjiang. In each region, the major indigenous group is dominant, but at the same time coexists with substantial numbers of Han Chinese and smaller

Abbreviations: CAM, complementary and alternative medicine; MSM, modern scientific medicine; TRM, traditional medicine; WHO, World Health Organization

'As will be made clear, what we are focusing on is longstanding and genuinely embedded forms of indigenous TRM, such as Tibetan, Uighur, and Mongolian medicine. This does not include forms of CAM that have been grafted on to healthcare systems, and often have few or no underpinnings in the society in which they are now being practised. We fully understand the scepticism with which such forms of medicine are often greeted by physicians, ${ }^{30} 31$ and we have no interest in saying how they might be integrated into established healthcare systems. ${ }^{32}$ The three forms of TRM under discussion are actually founded on the concept of "knowing practice" analysed by Farquhar ${ }^{33}$ and the forms of transmission examined by Hsu. ${ }^{34}$ Our concern is how these forms of TRM should be brought within contemporary policy frameworks. 
numbers of other indigenous peoples. In each region, the major indigenous group has an entrenched TRM. In Inner Mongolia, the local tradition is Mongolian medicine. In Tibet, Tibetan medicine is widely practiced, and in Xinjiang, Uighur medicine remains strong.

For many reasons, these regions form a good site for consideration of our core questions. First, the modern experience and current presence of the three TRM forms invite careful study of a significant issue in medical epistemology: how might we properly compare the effectiveness of TRM and MSM? This question is far more interesting in these three regions than in the Han Chinese heartland because the marginal status of indigenous TRM forms results from an imposed "scientific" evaluation in accordance with the MSM standard. Moreover, it is intellectually challenging to reexamine the dominant position that the only appropriate medical integration of TRM and MSM is to take MSM standards as fundamental. Is there solid epistemological evidence to support this claim? Is there any convincing reason why the medical legitimacy of TRM must be determined by MSM standards? Finally, as TRM remains an essential element of the indigenous ways of life in these regions, exploring these issues carries a significant practical implication. If the wishes and choices of indigenous people in living their preferred ways of life are to be respected, integrative medical systems in which the respective TRM systems find their proper places must be created.

The first main section of the article briefly surveys medical provision in Inner Mongolia, Tibet and Xinjiang. The next section explains the danger of fusing TRM into MSM. The subsequent section lays out the ideology of science that lies behind the demand that all traditional forms of medicine be evaluated and reformed according to MSM standards. The following two sections focus on improving TRM measures and comparing effectiveness, in both cases posing the question "whose standard?" The second of these two sections takes Tibetan medicine as a case study to indicate the falsity of an important premise of the scientific ideology, that the effectiveness of medical treatment depends on it conforming to the general and particular explanatory accounts of aetiology, pathogenesis and therapy found in MSM. The article concludes that the proper integrative system for TRM and MSM is a dual standard based form in which both TRM and MSM are free to operate according to their own medical standards.

\footnotetext{
iiTo summarise this complex history, four phases can be distinguished to indicate different official attitudes toward MSM and TRM integration in China. Phase I (from the mid-19th century to the collapse of imperial China in 1911) was a TRM standard based integration. Chinese medicine was taken to hold the correct foundations, purposes and principles of all medicines, and MSM treatments were only admitted as useful therapeutic means. ${ }^{35}$ Phase II (1911-1949) moved to the opposite position. The Nationalist government offered "unconditional support" to MSM, whereas TRM was taken to be a "feudal" practice or the "collected garbage of several thousand years" that should be prohibited from practice. ${ }^{36}$ Phase III (1950s-1980s) was marked by a Maoist campaign to create a "new medicine" through a combination of the best aspects of both Chinese and Western medicine. It attempted to produce a new standard based integration from mixed TRM and MSM standards, while being "fully scientific without being foreign or elitist" ". ${ }^{37}$ Phase IV (from the 1980s to now) is apparently characterised by a "three roads" policy. The drive to create a new medicine was substantially downgraded in the early 1980s. Officially, all TRM forms, MSM, and a conjunction of TRM and MSM have since been granted the freedom to exist. However, the three roads are not accorded equal importance. Rather, MSM has the upper hand, and TRM is required to prove its effective properties using the MSM standard. ${ }^{36}$ In this phase, TRM and MSM integration has thus taken an MSM standard based form.
}

TRADITIONAL MEDICINE IN INNER MONGOLIA, TIBET AND XINJIANG

Momentous Western engagement with Chinese civilisation, and the consequent meeting of MSM and TRM in China, dates from the mid-19th century. Although the history of engagement exhibited some differences in Inner Mongolia, Tibet and Xinjiang, the central themes were essentially common, especially after the creation of the People's Republic in 1949. Throughout China, modern medical history comprises the gradual domination of MSM in every region of the country. At the same time, the official attitude toward TRM and MSM integration has changed from the 19th-century insistence on TRM as the key standard of medical excellence to the more recent favouring of MSM as the core standard. ${ }^{\text {ii }}$

Nevertheless, TRM remains vibrant in all three regions. Particularly in rural areas, where most indigenous people live, TRM remains the folk medicine, and most people instinctively turn to TRM rather than MSM for healthcare. In the major cities, however, where indigenous people and Han Chinese cohabit and the top regional officials are based, the bulk of public investment in medical institutions has been poured into MSM facilities. In consequence, TRM has a much smaller profile than MSM. This is the case in all three regions and in all relevant areas: educational, clinical and pharmaceutical. The so-called "integrative system" is dominated by MSM in both quantity and quality, and TRM is only marginally present in the healthcare provision of leading medical institutions. As a result, a striking contrast exists in the three regions: the folk medicine is TRM, whereas the official institutional medicine is MSM.

\section{Context}

In 2000, Inner Mongolia had a population of 23.76 million, made up of both Han Chinese and Mongolians.. ${ }^{4}$ Mongolians numbered 3.97 million, making up about $17 \%$ of the total regional population. ${ }^{5}$ During the 20th century, modern Western medicine gradually came to dominate the region. Although Mongolian medicine has always been influenced by Chinese medicine, Indian medicines, and Tibetan medicine as practised in Buddhist temples, it has long existed as a separate tradition. ${ }^{6}$

In 2000, Tibet had a population of 2.62 million, ${ }^{4}$ with 2.41 million Tibetans, constituting about $92 \%$ of the regional population. $^{7}$ Although Tibetan culture is generally found in a purely Buddhist context, the extreme isolation of Tibet for much of its history means that its religion, philosophy and medical tradition have always had distinctive elements. ${ }^{8}$ In particular, they are characterised by a unique branch of Buddhism: Tibetan Tantric Buddhism.'

In 2000, Xinjiang had a population of 19.25 million, ${ }^{4}$ including about 8 million Uighurs, making up about $40 \%$ of the regional population. ${ }^{10}$ Uighur medicine traces its development across thousands of years, drawing partly on Ayurveda and traditional Indian medicine. However, the Silk Road connections to the Arab world and the West meant that Uighur medicine was also strongly influenced by ancient Arabic and Greek medicine.

\section{Education}

Traditionally, training in all three TRM systems was dominated by the master-disciple model. Since the 1950s, however, practitioners have been educated to the tertiary level in colleges established for this purpose. In Inner Mongolia, the Ethnic Medical College was set up in 1958, primarily to educate Mongolian medicine students. It is located in Tongliao, a city in the east of the region. There is also a Department of Mongolian Medicine at the Inner Mongolia Medical College in Hohhot, the capital city of the region, although this college primarily trains MSM students. Together, these two institutions graduate about 100 Mongolian medical practitioners each year from their 5- 
year bachelor's degree programmes. In contrast, at least 1000 specialists in MSM graduate every year from the region.

In Tibet, the first formal health school, the Lhasa School of Hygiene, was established in 1974 to provide training to the subdegree level. An independent School of Tibetan Medicine was created in 1983 and soon after, in 1985, the University of Tibet inaugurated a Department of Tibetan Medicine, offering undergraduate degree programmes. In 1989, the two institutions were merged to create the College of Tibetan Medicine in Lhasa. Annual graduates from the bachelor's degree programmes now average about 60 for the full 5-year programme, plus a further 35-40 from a top-up 3-year programme that upgrades sub-degree training to degree level. In comparison, 519 students were pursuing MSM degrees at the Tibetan University Medical College (the only MSM college in the region) in 2003. ${ }^{11}$ It is also important to note that a large number of MSM graduates and practitioners are recruited from other Chinese provinces into Tibet (especially Lhasa) to staff the region's MSM hospitals.

Training in Uighur medicine takes place mainly through the College of Uighur Medicine established in 1987 in Hetian, a city in the west of Xinjiang. In total, 1200 graduates have since been produced. There are currently about 1400 students on campus, plus about 200 students in hospitals gaining practical training. In contrast, Xinjiang Medical University in Urumqi, which trains both MSM and Chinese medicine practitioners, produced 994 graduates in 2003 and currently has 17216 students on campus. $^{12}$

\section{Hospitals}

In Inner Mongolia, hospitals providing Mongolian medicine are limited in the number of beds and physicians. In 2003, the region had 31 Mongolian medicine hospitals, with 968 beds in total; 55 Chinese medicine hospitals, with 4192 beds in total; and 4 combined MSM and Chinese medicine hospitals, with 295 beds in total. ${ }^{13-16}$ However, the region had 472 hospitals in total, ${ }^{17}$ thus there were $>360$ MSM hospitals, with $>50000$ beds.

Across the region of Tibet, there are 17 Tibetan medical hospitals, plus a multitude of clinics and small-scale freelance practitioners in rural areas. Of the 17 Tibetan medical hospitals in the region, the main hospital, established in Lhasa in 1916, belongs to the autonomous region and forms the focal point for the practice of Tibetan medicine. In 2003, it had approximately 250 beds. The remaining 16 hospitals belong to lower levels of administration. In 2003, these 17 hospitals had 552 beds in total. ${ }^{14}$ However, the region had 97 hospitals in total ${ }^{17}$, thus there were 80 MSM hospitals, with $>5000$ beds.

In Xinjiang, there were 41 hospitals that specialised in Uighur medicine in 2003, with 2109 beds. ${ }^{14}$ However, in total, the region had 682 hospitals $^{17}$ thus there were $>600$ MSM hospitals, with $>60000$ beds.

\section{Pharmacy}

Although the major TRM hospitals in all three regions continue to produce many drugs on site, additional factories have recently been built to expand production and spearhead a marketing drive that aims at the growing market in other parts of China and overseas. Furthermore, many compounds use modern methods of presentation, in the form of tablets and capsules. A critical factor is national accreditation to overcome the concerns about quality that have plagued traditional compounds for many years. The Chinese government started work on this in 1998. In Xinjiang, in the 3 years to the end of 2000, 30 of about 1000 different Uighur medical products (of which about 400 are in regular use) were accredited, and this accreditation effort is continuing.
Meanwhile, there has been a great deal of interest in "modernising" traditional drugs. In all three regions, new companies have been set up to reform these drugs without relying on traditional methods and understanding. Instead, they seek to exploit modern theories to explain the active ingredients and properties of their drugs so as to produce modern medications by abstracting effective chemical elements from traditional prescriptions. This tendency appears to be accelerating in each region.

\section{FROM MARGINALISATION TO FUSION}

It is clear that MSM is now stressed in the integrative systems of Inner Mongolia, Xinjiang and Tibet, although TRM is active in the ordinary medical practice undertaken in clinics or by individual practitioners in villages and small towns. In all areas, MSM has been given priority over TRM, and TRM has been virtually pushed to the margins of the healthcare system. Moreover, in addition to its small size, TRM faces an identity crisis in each region; TRM is evaluated in the Weberian sense as "disenchanted," and reformed according to the MSM standard. It is therefore increasingly difficult for each TRM to stand independently, free from the intervention and amendments of MSM designed to change its substance. These tendencies are driving not only the marginalisation of TRM, but also a fusion of TRM into MSM.

Indeed, to distinct degrees in all three places, experiments in medical fusion are taking place, integrating MSM into indigenous medical theories and practices. Furthermore, the fusions run in all possible directions. In education, TRM colleges have invested greater amounts of time and resources into teaching MSM and related modern scientific theories and technologies, rather than focusing on TRM classics (in the case of Tibetan medicine, for example, the Four medical tantras) and techniques. Traditional practitioners are now required to know a great deal of modern medicine, though the reverse is not the case. In research, TRM researchers tend to choose more "scientific" topics for their research projects, and graduate students prefer more "scientific" scholars as their supervisors. In the pharmaceutical sector, for instance, not only are advanced modern technologies and facilities now being used to manufacture traditional prescriptions and drugs, but also research projects are designed and conducted under the direction of modern scientific theories to discover effective chemical ingredients from traditional drugs and prescriptions.

In clinical practice, reforming TRM has been the dominant attitude of healthcare administrators, who implicitly or often explicitly value MSM over TRM. TRM hospitals have equipped themselves with advanced modern Western diagnostic and therapeutic facilities in order to "scientificise" themselves and compete with MSM hospitals. TRM hospitals are also divided into different departments according to the MSM standard, and often offer much the same range of services as MSM hospitals. Perhaps the only significant difference is that in a TRM hospital the patient will, in addition to receiving conventional MSM drugs, also receive indigenous medicaments for treatment.

Indeed, TRM physicians in such TRM hospitals usually administer "double diagnosis" and "double therapy" in their practice. For every patient they make two diagnoses: one according to TRM explanations and another according to MSM theories. They then prescribe both TRM compounds (based on the TRM diagnosis) and MSM chemical drugs, or they conduct other MSM therapies (based on the MSM diagnosis). As a result, although MSM physicians only conduct MSM therapy, TRM physicians take double therapy for granted. This practice has strengthened the popular impression that, for most medical problems, MSM should do the main work, although TRM may offer some minor, complementary assistance. 
In evaluation and regulation, the scientific standard of MSM is used to evaluate every aspect of TRM. In addressing TRM, the government has emphasised "scientificisation" (kexuehua), created through "scientific" research, "scientific" explanations and the "scientific" reorganisation of TRM. Such policies have caused enormous difficulties for TRM physicians, especially young physicians applying for promotion, to practice TRM in official medical institutions. They often find themselves facing a review committee consisting mainly of MSM experts. These experts are either unable or unwilling to evaluate TRM physicians solely according to TRM standards. Instead, TRM physicians are often evaluated according to the extent to which they have grasped and practiced MSM procedures and measures. Moreover, TRM physicians are afraid that if they are subject to a malpractice lawsuit, the assessment norms will primarily be drawn from MSM. This has induced them to adopt more MSM than TRM in their practice.

\section{THE IDEOLOGY OF SCIENCE}

What has caused the asymmetry between the popularity of folk TRM practices, and the institutional marginalisation (or fusion into MSM) of TRM? The answer lies primarily in the ideology of science that has been adopted by the Chinese authorities to evaluate all forms of medicine. This ideology supports the general view that, if one medical approach (that is, MSM) is more scientific and more effective than another, then in an integrative system the less scientific and less effective approach should be improved according to the standards of more scientific and more effective approach. In this ideology, MSM approaches are those that, inter alia, appeal to methods of correcting observer bias (eg, statistical methods) in order to demonstrate their greater predictive capacities. Also in this ideology, a medical approach is more effective if it can be demonstrated through appeal to methods for correcting observer bias (such as statistical methods) that it more reliably lowers risks of morbidity and mortality than other medical approaches. Moreover, the greater effectiveness of MSM is held to be due to its causal accounts of disease and therapy, which allow it to be more effective. This ideology can be condensed into the following argument:

- The more scientific, the more effective (premise 1).

- MSM is more scientific than TRM (premise 2).

- Therefore, (a) MSM is more effective than TRM, and (b) in the integrative system of TRM and MSM, TRM should be improved according to the MSM standard so as to become more scientific and therefore more effective. ${ }^{\text {ii }}$

Conclusion (a) explains why MSM has become predominant in medical institutions in all three regions. Conclusion (b) explains why TRM has increasingly been fused into MSM.

Logically, we could block the two conclusions by rejecting premise 2. For instance, some Chinese have tried through coining a special understanding of science to contend that TRM is as scientific as MSM, or even more scientific than MSM. ${ }^{\text {iv }}$ However, this strategy is nothing more than quarrelling about the conventional modern understanding of science. Like it or not, the modern sense of science is now solidly established, with a series of arranged empirical knowledge and rules regarding observation, evidence and inference. They have been

iiilt should be noted that the ideology of science has never been explicitly expressed in an official Chinese document as it is summarised in this argument. However, premise 1 has generally been accepted by both the government and society. Premise 2 is controversial in academic discussions, but the opposite view barely has any practical influence. In addition to being supported by the two premises, the conclusions have been carried out in the medical practice in China. widely adopted and manifested in the core disciplines of modern science, such as physics, chemistry and biology. Because this sense of science has been broadly accepted in modern China, challenging premise 2 is equivalent to offering a new definition of science, which is at best missing the point of the issue being debated.

Alternatively, we could block the two conclusions by rejecting premise 1 , which is indeed what this paper intends to do. We believe that premise 1 cannot stand. Moreover, because it is the crux of the scientific ideology that has directed the shaping of inappropriate integrative systems in the three regions, it must be rejected in order to return the major indigenous medicines to their proper places. Before explaining how and why we refute premise 1 , a particular issue generated by conclusion (b) will be addressed in the next section.

\section{IMPROVING TRADITIONAL MEDICINE: WHOSE STANDARD?}

Conclusion (b) states that TRM should be improved according to the MSM standard so as to become more scientific and thereby more effective. The issue is, if TRM has become more scientific in this way, is it still TRM in nature? Or has it become a part of MSM? In short, is it possible to improve TRM according to the MSM standard without changing the nature of TRM? We hold that the answer depends on which of two different types of improvement are made to TRM.

The first type is an MSM standard that inspires or suggests a reform or revision of a particular TRM measure or practice, while allowing the reform or revision to be accredited by the TRM standard. For instance, the method of using alcohol to sterilise acupuncture needles is learned from MSM, but it can readily be accredited by the TRM standard because TRM is also concerned with cleaning the needles and can easily accept a more effective way of doing it. ${ }^{\mathrm{v}}$ Another good example is the use of modern technologies and facilities to manufacture TRM drugs in more efficient ways. In this sense, a medical system does learn and can benefit from the methods of a different medical system, and can gain improvement according to its own standard, even though distinct medical systems operate on parallel lines defined by their unique theoretical underpinnings. This reveals the complexity of mutual medical learning. In theory, it is difficult, indeed largely impossible. In practice, however, it can be helpful for physicians to draw insights and borrow measures from more than one tradition. The crucial point is that the newly learnt measure should be incorporated into its own system of medical standard.

The second type occurs when MSM therapies and strategies are forced on TRM, but cannot be accredited by the TRM standard. A typical example is attempting to find and abstract effective chemical ingredients from TRM prescriptions in order to make MSM medications. This already constitutes a special MSM activity and can be advocated by the MSM standard. However, because such an activity is also incommensurable with basic TRM theories and principles regarding prescriptions and medications, it cannot be accredited by the TRM standard.

iv In mainland China, such arguments are readily heard. For instance, there is a contention that TRM is more scientific than MSM because TRM holds a holistic view, whereas MSM holds an atomistic and reductionist view. Such an argument does not possess solid intellectual strength because the key concepts appealed (such as holism and atomism) are often unclarified.

'Is this also accredited by the MSM standard? Seemingly, yes, because this is consistent with the MSM requirement of cleaning any medical instrument used in contact with the body. However, if we take into account the fact that the practice of acupuncture cannot make sense in the MSM system, and thereby cannot be accredited as a therapeutic practice by the MSM standard, the answer would have to be "No". 
Although it is legitimate for MSM to conduct such activities in order to develop its pharmaceutical industry, the medications thus produced are indeed MSM drugs, not TRM drugs. Hence, instead of seeing such activities as successful "improvements" of TRM, they should be seen as MSM activities in nature. If a TRM uses them to replace its original medical prescriptions and productions, it would only imply the dissolution or fusion of that TRM into MSM.

\section{COMPARING EFFECTIVENESS: WHOSE STANDARD?}

Once premise 2 (MSM is more scientific than TRM) is granted, only premise 1 (the more scientific, the more effective) offers any hope for derailing conclusion (a) (MSM is more effective than TRM). If premise 1 holds, the dominant position of MSM in the integrative systems of the three regions is warranted. Unless it can be challenged, the fate of TRM in these regions will inevitably be doomed.

Is it true that the more scientific a medical measure, the more effective it is? In particular, is it true that MSM is more effective than TRM? A careful elaboration on the concept of effectiveness is required. First, it is essential to recognise that "effectiveness" is a value-laden term. Saying that A is effective for B is not simply stating that A constitutes a sufficient condition for B, but also establishes B as a goal to be pursued by A. For instance, if eating an appropriate, regular breakfast is taken to be effective for maintaining health, it implies that health has been established as an end to be pursued through eating breakfast. This is to say that judgements about effectiveness or efficacy entail making goalrelevant or expectation-dependent statements, and such goals or expectations manifest the values that have been ordained in the "life world"-namely, people's lived experience. If nothing has been established as an end, then nothing can be stated as effective or ineffective as a means. Accordingly, the medical activities that people are engaging in to judge "effectiveness" or "efficacy" have not only assumed the necessity of a cause-effect mechanism in the natural world, but have also assigned the necessity of an end-means structure to the life world.

In order to disclose an end hidden behind a medical judgement that a particular treatment is effective, it is necessary to ask the question "effective for what?" There is an age-old cliché that health is the end of medicine. Some may want to contend that no matter which medical tradition is concerned, the goal of medicine is always to preserve health, prevent disease and treat illness. This contention is formally true. However, it cannot be substantively true because different medical traditions have developed different substantive goals expressed in their various, incompatible understandings of health, disease and illness. That is, they do not understand health, illness and disease in similar ways, and by similar we mean mutually commensurable. Accordingly, although distinct medical traditions share the same formal goals of preserving health, preventing disease and treating illness, this formal goal is useless in comparing the effectiveness of different medical traditions. Evidently, every long-standing medical tradition has developed within a particular culture: its key concepts of disease, illness and health, as particular substantive concepts, have functioned within a net of specific goals granted in that culture, based on its particular evaluative and explanatory assumptions. Accordingly, a medicine is best taken to be part of the function of a particular culture. In order to decide what is a suitable medical problem and what is an appropriate medical way to deal with the problem, a distinct group of substantive goals set in a particular culture must be referenced. ${ }^{18}$

Indeed, distinct medical traditions have set up different goals and expectations based on their unique, underpinning religions and philosophies. ${ }^{\mathrm{vi}}$ They each hold a complex matrix, which contains not only a distinct evaluative system (particular goals and expectations to pursue), but also a specific explanatory framework (empirical rules to follow). Their different conceptual frameworks lead to different empirical systems, expressed in particular values and goals and in distinct rules of evidence and inference, which may overlap but never coincide with each other. In this sense, different medicines are incommensurable with each other in both concept and experience. To demonstrate this point in a concrete manner, we turn to Tibetan medicine as an illustrative example to show how it holds different and incommensurable substantive goals (manifested in its views of prevention, treatment and pharmacy) from those of MSM.

\section{Preventive goals}

Tibetan medicine has been significantly influenced by the Buddhist view of world and life. ${ }^{\text {vii }}$ Disease is seen as a type of suffering. Suffering is not accidental but springs from the fact that all creation is transitory and all phenomena are impermanent. According to the special aetiology offered by Tibetan medicine, all diseases are ultimately caused by karma (a person's negative actions), including karma from previous lifetimes and karma obtained at an earlier period of this life. ${ }^{19} 20$ Accordingly, the Buddhist sees that the fundamental way of preventing diseases lies in eschewing negative actions by seriously living a Buddhist way of life.

A person's karma is ultimately due to an ego manifested in the form of ignorance (that is, the ignorance of believing that there is such a thing as a truly existent self). This ignorance leads to the three poisons: attachment, hatred and closemindedness. These three poisons in turn give rise to the afflictions of the three humors in the body (Air, Bile and Phlegm) so that their balance is broken and disease occurs. ${ }^{19}$ This understanding sets forth a close interrelationship between the physical (the humors) and the mental (attachment, hatred and close-mindedness), which is totally alien to MSM. Extinction of suffering means liberation from the vicious cycle of existence, and this is accomplished through the proper knowledge and genuine practice of the Dharma, the religion that protects persons from disasters. ${ }^{21} 22$ In particular, in order to prevent disease, Tibetan medicine offers detailed recommendations on everyday affairs and sacred regimens for individuals to observe. ${ }^{19} 2324$

This is not to say that Tibetan medicine does not hold a cosmology consisting of basic physical elements. Instead, it holds that everything is composed of the five cosmic elements or energies: earth, water, fire, air and space. It is roughly correct to state that it is on these five energies that Tibetan medical anatomy, pathology and pharmacology are established. The five basic energies influence the body through the humors: air (wind)

vilooking back through history, three great strands of medical practice provide the ultimate foundations for the various forms of medicine found in the world today: Chinese, Greek and Indian. The conceptual bases of all three traditions are fundamentally at variance with each other. Beyond that, subsidiary medical traditions have also developed, often based on subtle religious and philosophical distinctions that set them apart from the mainstream that originally inspired them. Mongolian, Tibetan and Uighur medicines belong to the latter category.

\footnotetext{
viiThe cardinal classic of Tibetan medicine, Four medical tantras (compiled in the 8th century, comprising four treatises: the Root Treatise, the Explanatory Treatise, the Practice Treatise, and the Appendices Treatise) can reasonably be taken as using basic Buddhist principles to reorganise all existing medical materials, including local Tibetan practices and practices learnt from Indian and Chinese medicines. For information about the source of this classic, see Rinpoche (2001) pp. 3-4; Cai (2002)pp. 5470 and Qiangbachilie (1996/ pp. 1-18. ${ }^{38-40}$ We have not found a reliable complete translation of the book into English; for a Chinese translation, see Li (1983). ${ }^{19}$
} 
affect Air; fire (heat) affect Bile; earth and water (dampness and humanity) affect Phlegm. When these energies are not well balanced, the humors are disturbed and disease occurs. ${ }^{25}$ However, it is totally mistaken to understand these energies and the process of their interactions merely in terms of a materialist vision. The type of Buddhism on which Tibetan medicine is solidly based is Tantric, a highly practical form of mysticism affording precise techniques for attaining that wisdom whereby the person's ego is negated and they enter upon the bliss of their own divinity. Specifically, Tantric Buddhism holds that the five elements have three levels: gross, subtle, and essence aspects. The essence aspect is a pure mind, identified and operated by the Tantric "Wisdom Mind". ${ }^{26}$ This aspect profoundly informs Tibetan Buddhist therapy and pharmacy.

Interestingly, for Tibetan Buddhism, an attempt to liberate oneself from the vicious cycle of existence by no means implies that one must live an ascetic or depressed life. To prevent disease, it is crucially important to follow the physiological desires rather than hold or suppress them:

\section{Do not obstruct the impulses of hunger, thirst, vomiting, yawning, sneezing, breathing, sleeping, to clear mucus from the throat, to remove excess saliva from the mouth and throat. Do not suppress the desire to defecate or urinate, do not hold or suppress intestinal wind and gas or block the emission of semen... By obstructing (or alternatively forcing these actions), all ailments arise and the winds are immediately disturbed. ${ }^{19}$}

In addition, the symbolism of Tantric Buddhism is the unity of the male and female energies manifested in a positive approach to sexuality: some seemingly erotic forms of sexual intercourse are freely used for conveying religious feelings and for pursuing health. Not only does sexual intercourse have no evil associations (no implications of licentiousness), but also it has a positive value for health. ${ }^{27}$

\section{Effective treatment}

Substantive Tibetan medical therapeutic goals are embedded in the specific Tantric Buddhist convictions on which Tibetan medicine stands. First, Tibetan medicine distinguishes two levels of treatment: fundamental and mundane. The fundamental treatment is the Buddha's three cures: Wisdom-insight cures ignorance and confusion; Virtue-compassion cures attachment, clinging, greed and lust; and Contemplation-meditation cures anger, hatred and aggression. ${ }^{27}$ Mundane treatment uses medications and other ordinary medical techniques.

It is not the case that doctors only appeal to mundane treatment, leaving fundamental treatment to the Buddhist priests (gurus). Instead, a good physician is required to provide complete treatment. In order to offer complete treatment, the physician's mind cannot stay only at the gross and subtle element levels of the patient's body. From the Tantric "Wisdom Mind" view, unless the doctor's mind is able to appreciate and apply itself on the essence element level, the resulting treatment will only be partial or superficially complete, diagnosis incomplete or symptomatic rather than fundamental and correct, and the clinical result most probably temporary. ${ }^{27}$ In short, a real doctor must be able to go beyond the gross and subtle element aspects of the patient's body in order to see the fundamental spiritual (mind) cause of the problem and thereby conduct proper treatment. ${ }^{28}$

This requires doctors to grasp certain delicate techniques. A prominent example is Tibetan medical pulse examination. Pulse diagnosis is a basic tool through which the doctor can identify and evaluate the deepest spiritual (not only the general) causes of the humoral imbalance of the patient. ${ }^{27}$ Various complicated phases of pulse have been established in
Tibetan medicine for doctors to grasp, such as death pulse, evil spirit pulse, lifespan pulse, guest pulse, family pulse, enemy pulse, friend pulse, reversing (role) pulse, and so on. To master such distinct pulse phases is not only a highly technical, longterm experiential matter, ${ }^{28}$ but it is also necessary to appreciate the Tibetan Buddhist world view and its fundamental values. For instance, family pulse implies that a close family member of the patient can substitute the examination of his or her pulse for the patient. This is significantly "effective" when the patient cannot be examined directly by the physician due to the limitation of the circumstance (such as the patient is too sick to go to see the doctor). Tibetan medicine holds that the imbalances in the humors of the patient can be read on the relative's pulses given that they are closely related people. ${ }^{27}$

\section{Effective drugs}

Preparing Tibetan medications for exerting their effects is not solely pharmaceutical in the modern sense. Instead, Dharma and Tantric ritual performance (such as reciting prayers) must be present throughout the entire process of preparation. Such ritual preparation is intrinsic and fundamental to the preparation of Tibetan medicinal compounds, including the wellknown prophylactic Tibetan medicines, such as Tsothel and other precious pills. ${ }^{27}$ Such rituals are taken to empower the medicines to become "animate from the inanimate," and to enhance the curative powers of the medicinal ingredients. They constitute the very pith and kernel of the Tantric Buddhist basis of Tibetan medicine with its three stages:

\section{Giving the medicines empowerment directly; \\ Visualising the medicines as the deity; \\ Visualising one's self and the medicines as the deity. ${ }^{27}$}

In Tibetan medicine, the pharmaceutical processes deal only with the gross element aspects that influence the gross somatic Five Element matrix of the physical body, whereas the ritual preparation is concerned with the pure essence of the medicines. Accordingly, the pure essence of the preparations treats on the pure essence level (mind) of the patient. ${ }^{28}$ Some may want to contend that this is not objective because it sounds like psychic means, playing a placebo effect. However, such ritual preparation of drugs is obtained from the ancient Tantric "Wisdom Mind" view, which has always been an inseparable, indeed a central part, of the Tibetan medical tradition. Even if its role is only religious or psychological rather than medical in the modern scientific sense, it is still a unique contribution to patients' healthcare. In any case, practitioners of any school of medicine have great difficulty in determining the degree of psychosomatic element in the majority of patients' conditions.

\section{Incompatible goals}

The above discussion shows that the substantive goals of Tibetan medicine contrast sharply with those sought by MSM. Tibetan medicine holds that the Tantric Buddhist principle of the essence aspect is involved in all creatures and operated and identified through the "Wisdom Mind." This belief significantly directs and shapes the specific Tibetan medical goals. In prevention, the goal includes avoiding karma (negative actions), discharging physical desires smoothly, and seeking the proper union of man and woman in sexual ways. ${ }^{\text {viii }}$ In treatment, the goal involves fulfilling complete therapy by pursuing fundamental rather than

\footnotetext{
viiiititual treatment is taken to be very effective in Tibetan medicine. A prominent example is regular kneeling in prayer. Differing from other prayers in which one kneels only a few times, the Tibetan way is to do it hundreds or thousands of times. Although this is primarily a religious activity, it is also taken as a very effective medical treatment, especially for digestive diseases (see Ga (1996) p. 156). ${ }^{41}$
} 
mere mundane treatment. In pharmacy, the goal covers enhancing the effect of medication through Tantric rituals. Seeking these specific goals leads to a comprehensive positive ideal of health in the Tibetan Buddhist way of life. ${ }^{\text {ix }}$ As a result, Tibetan medicine contains a system of end-means structures that is entirely alien to and incommensurable with that of MSM. Accordingly, when MSM physicians see some Tibetan medical interventions as ineffective, they may simply make the judgement in terms of the substantive ends of MSM, while being ignorant of the particular goals that Tibetan medicine pursues. For instance, if one claims that the Tibetan medical attempt to improving the humor disturbance of a terminally ill patient by examining his family member's "family pulse" is less effective than the MSM palliative care, one must overlook the fact that a goal of Tibetan medicine is to offer fundamental treatment regarding the patient's essence aspect, which is closely related to the essence aspect of his family members. This is to say, given that Tibetan medicine does not hold the same set of substantive medical ends or goals as MSM, it is not possible to state that MSM is more effective than Tibetan medicine, or vice versa. As substantive medical goals indicate concrete values that a medicine is pursuing through certain mechanisms and means, then unless we can judge that the substantive goals of MSM are more appropriate than those of TRM in promoting health, preventing disease and curing illness in the formal sense, we cannot claim that MSM is more effective than the TRM. Comparing TRM and MSM is ultimately comparing their incompatible substantive medical goals.

Can we tease out a set of "neutral" substantive goals for evaluating MSM and TRM? Some may want to argue that indices such as life expectancy, morbidity and mortality rates in general, and infant and maternity mortality rates in particular, plus emergency rescue success rates and other indices can be used as neutral standards to compare the effectiveness of MSM and TRM. That is, they may contend that both MSM and TRM should accept these indices as fair criteria to evaluate their respective medical practices, even if they hold incompatible substantive medical goals. However, the difficulties with such a "neutral" standard strategy are multiple. First, many factors other than medicine contribute to the variation of these indices. An effective medicine is at best a necessary, but not sufficient, condition for good scores on these indices. Second, there is the issue of discrepancy between theory and practice. Suppose medicine $\mathrm{P}$ is in theory more effective than medicine Q (meaning that, all other things being equal, a set number of doctors using $\mathrm{P}$ will generate better indices than the same number of doctors using Q). However, the reality may well be that doctors using Q do more effective work than doctors using $\mathrm{P}$ because the latter have not received such good training and proper regulation as the former, even though their medicine is theoretically superior (indeed, this was the complaint of many TRM physicians in the three regions during our investigation). Third, the values manifested in these indices may not be evenly promoted by each medicine. Today, many people believe that TRM is better than MSM in promoting longterm health and thereby increasing average life expectancy, whereas MSM is better than TRM in critical care, especially for the very elderly. Suppose this belief is true. ${ }^{\mathrm{x}}$ Then, judging which medicine is more effective will depend on which index is taken to be more important: average life expectancy or critical care? Another good example in this regard is orthopaedics. All three

${ }^{i x}$ According to Tibetan medicine, health has two flowers and three fruits. The first flower is freedom from disease, the second long life. The first fruit is Dharma, including Worldly Dharma (the development of noble human characteristics) and Divine Dharma (the following of the Buddhist religious path). The second is wealth, including both material and spiritual wealth. The third is happiness, having the capacity to free oneself from confusion and ignorance and attain liberation (Enlightenment). ${ }^{27}$
TRM systems we have investigated have superb orthopaedists who are capable of quickly and accurately setting broken bones by hand without the need of appealing to surgery or using complicated modern technological facilities. The patients can immediately return to their homes and recover quickly. The only problem may be that this involves rather more pain than the parallel MSM treatment. Accordingly, whether we favour such traditional bone-setting methods or MSM orthopaedics depends on how we trade off between an experience of pain plus somewhat faster recovery versus no experience of pain plus somewhat slower recovery. There are also additional medical and social implications; for instance, TRM methods are usually much less expensive than MSM interventions.

Finally and most significantly, the values manifested in these indices cannot always be pursued consistently with other human values involved in medical practice. For instance, all three TRM systems have developed useful knowledge and skills in aiding baby delivery at home, fitting well into the local religious beliefs in favour of home delivery. It may be the case that no matter how perfect such home delivery techniques become, home delivery will still involve slightly higher rates of infant and maternity morbidity and mortality than delivery at a well-resourced MSM hospital. However, in this case, the effectiveness of a medicine can no longer be judged only in terms of the "neutral" values of the indices. If people take it to be fundamentally important to deliver at home (for they may want to hold a religious ritual that can only proceed at home, in addition to cherishing the mother's psychological comfort in the company of her family members in a familiar environment), the most effective medicine will be the one that can offer most assistance at home rather than in hospital.

This leads us to recognise that a medicine functions within a particular culture. Because different cultures hold different and mutually incommensurable goals, they may require the use of different and mutually incommensurable means, among which are medical means. Even if all medicines have the same formal goal of pursuing health, they differ from each other in their substantive goals regarding what health is and how health should be pursued. The "neutral" medical indices cannot indicate which medicine is more effective because, among other things, the values manifested in these indices have to be balanced with each other as well as with other values pursued in a particular culture. Different medicines do not behave evenly with regard to the various configurations of such values. Accordingly, the effectiveness of MSM is only embedded in certain cultures with certain substantive values and goals, not in other cultures pursuing different substantive values and goals. In this sense, premise 1 (the more scientific, the more effective) is false.

\section{CONCLUSION}

Different forms of medical integration have been attempted in modern Chinese history: TRM standard based, MSM standard based, and new standard based. The Chinese have broadly recognised the failure of the TRM standard based integration (primarily in the 19th century) and the defect of the new standard based integration (primarily in the mid 20th century). If this article's argument is sound, then the currently dominant MSM standard based integration is also indefensible. Regions with vibrant established TRM systems (such as Tibet, Inner

${ }^{x}$ Some TRM experts do not agree with this assumption. For instance, an expert from the Tibetan Medical School told us that the current model of practice is problematic. In Tibet, integration at the clinical level takes place particularly when a physician is faced with a critical situation. In such cases, MSM may well be used initially to stabilise the patient, before switching to Tibetan medicine for longer-term care. The expert argued that the effective critical care techniques of Tibetan medicine have been curbed from application and development by this model of integration. 
Mongolia and Xinjiang) should change to a dual standard based system whereby TRM and MSM operate independently according to their own standards. ${ }^{1}$

To be fair, the Chinese authorities have made efforts to preserve the indigenous medical sectors in the medical institutions of all three regions. They are staffed by highly skilled practitioners, and overseen by deeply committed and able officials in the State Administration of Traditional Chinese Medicine and its regional and local offices. However, the ideology of science is all-pervasive, and thus disenchants practitioners of TRM. MSM is simply too dominant, and TRM is in retreat. In order to reframe proper integrative systems in these regions, greater attention needs to be paid to ensuring that modern and traditional medicines are able to function on different but equal bases. As each is founded on unique conceptual underpinnings carrying particular values and goals and distinct empirical rules, it becomes difficult to hold that a single measure could ever be found to evaluate competing traditions. In this domain, as in so many others, there is no universal standard, and a positivistic search for the "evidencebased high ground" is likely to be counterproductive. ${ }^{29}$ The choice then is either to arrange medical traditions in a hierarchy and distribute status privileges among them, or to set them on equal bases. We know of no good argument for building a hierarchy, and can think of many valid reasons why setting medical traditions on an equal footing should be the approach taken. ${ }^{2}$

In all three regions, this requires significantly enlarging the size of TRM in recently developed medical institutions in urban areas, including educational, pharmaceutical and clinical. For instance, although it is clearly helpful for each region to fund specialist TRM hospitals, it would also make sense for every large-scale, comprehensive MSM hospital to contain some departments offering traditional therapies. Thus, mutual referral between modern and traditional medical practitioners in these regions can become more effective. Finally, TRM systems must be regulated based on their own standard, rather than the MSM standard. This is also our prescription for policy frameworks in other societies with established, indigenous medical sectors.

\section{ACKNOWLEDGEMENTS}

The research reported in this article was supported by a grant from City University of Hong Kong (Project No. 7100257). We thank officials and practitioners in Inner Mongolia, Tibet and Xinjiang for talking to us about traditional medicines. We are also very grateful for the incisive and constructive criticism from the two reviewers appointed by this journal, Professor Griffin Trotter and Professor H. Tristram Engelhardt Jr. The usual disclaimer applies.

\section{Authors' affiliations}

R Fan, Department of Public and Social Administration, City University of Hong Kong, Kowloon, Hong Kong

I Holliday, Faculty of Social Sciences, The University of Hong Kong, Hong Kong

\section{REFERENCES}

1 Fan R. Modern western science as a standard for traditional chinese medicine: a critical appraisal. J Law Med Ethics 2003;31:213-221.

2 Holliday I. Traditional medicines in modern societies: an exploration of integrationist options through East Asian experience. J Med Philos 2003;28:373-89.

3 World Health Organization. WHO traditional medicine strategy 2002-2005. Geneva: WHO, 2002.

4 National Bureau of Statistics, People's Republic of China. Communique on major figures of the 2000 population census, 2001.http://www.cpirc.org.cn/ en/e5cendata2.htm (accessed 19 February 2006).

5 Inner Mongolia Autonomous Region. (In Chinese) http://www.china.org.cn/ chinese/zhuanti/166208.htm (accessed 19 February 2006).

6 Qigeqitu. Modern mongolian medicine (Xiandai Mengyixue). Liaoyang: Liaoning Minzu Press, 2002.

7 Xizang Autonomous Region. (In Chinese.) http://www.china.org.cn/chinese/ zhuanti/166235.htm (accessed 19 February 2006).

8 Kapstein MT. The Tibetan assimilation of Buddhism. New York: Oxford University Press, 2000.
9 Blofeld J. The tantric mysticism of Tibet. Boston: Shambhal, 1987.

10 Xinjiang Uygur Autonomous Region. (In Chinese. ) http://www.china.org.cn/ chinese/zhuanti/166324.htm (accessed 19 February 2006).

11 Tibet University. http://www.utibet.edu.cn/cn/ structures.jsp?flag = ???? \&menuld = 3 (accessed 19 February 2006).

12 Xinjiang Medical University. (In Chinese) http:// www.daxue.learning.sohu.com/school_moreinfo_382.html laccessed 19 February 2006).

13 Figures of TRM Hospitals in each province provided by the State Administration of Traditional Chinese Medicine of the People's Republic of China. http:// www.satcm.gov.cn/lanmu/atog/2003/b25.xls (accessed 19 February 2006).

14 Statistics of TRM Hospitals in each province provided by State Administration of Traditional Chinese Medicine of the People's Republic of China. http:// www.satcm.gov.cn/lanmu/atog/2003/a32.xls (accessed 19 February 2006).

15 Statistics of Traditional Chinese Medical Hospitals in each province provided by State Administration of Traditional Chinese Medicine of the People's Republic of China. http://www.satcm.gov.cn/lanmu/atog/2003/a28.xls (accessed 19 February 2006).

16 Statistics of Integrated MSM and TCM Hospitals in each province provided by State Administration of Traditional Chinese Medicine of the People's Republic of China. http://www.satcm.gov.cn/lanmu/atog/2003/a30.xls (accessed 19 February 2006)

17 Statistics of Healthcare Institutions in each province provided by the Ministry of Health of the People's Republic of China. http://www.moh.gov.cn/ openstatistics/year2004/p4.htm (accessed 19 February 2006).

18 Engelhardt, Jr, HT. The foundations of bioethics, 2nd ed. New York: Oxford University Press, 1996.

19 Li Y. Four medical tantras (Si Bu Yi Dian) (Translation. ) Beijing: People's Health Press, 1983;46.

20 Khangkar D. The Buddhist way of healing. New Delhi: Lustre Press, 1998:43.

21 Dalai Lama. The Buddhism of Tibet (Translated by Jeffrey Hopkins. ) Ithaca: Snow Lion Publications, 1987:23.

22 Tsarong J. Fundamentals of Tibetan medicine. Dharamsala: Tibetan Medical Center, 1981;(India).

23 Sachs R. Tibetan Ayurveda: health secrets from the roof of the world. Rochester, VT: Healing Arts Press, 2001.

24 Dash VB. Tibetan medicine: theory and practice. Delhi: Indian Books Centre, 1997:69-79.

25 Donden Y, Tshering G. An introduction to Tibetan medicine. New Delhi: Tibetan Review Publications, 1976.

26 Guenther H. The concept of mind in Buddhist Tantrism. In:Guenter H.Tibetan Buddhism in western perspective. Berkeley: Dharma Publishing, 1989:36-59.

27 Dummer T. Tibetan medicine and other holistic health-care systems. New Delhi: Paljor Publications, 1994.

28 Khangkar LD. Lectures on Tibetan medicine. Dharamsala: Library of Tibetan Works and Archives, 1998.

29 Richardson J. Evidence-based complementary medicine: rigor, relevance, and the swampy lowlands. J Altern Complement Med 2002;8:221-3.

30 Shine KI. A Critique on complementary and alternative medicine. J Altern Complement Med 2001;7:S1 45-52.

31 Furnham A, McGill C. Medical students' attitudes about complementary and alternative medicine. J Altern Complement Med 2003;9:275-84.

32 Barrett B. Alternative, complementary, and conventional medicine: is integration upon us? J Altern Complement Med 2003;9:417-27.

33 Farquhar J. Knowing practice: the clinical encounter of Chinese medicine. Boulder, CO: Westview Press, 1994

34 Hsu E. The transmission of Chinese medicine. Cambridge, UK: Cambridge University Press, 1999.

35 Ma B, Gao X, Hong Z. History of debate between traditional Chinese medicine and modern scientific medicine (Jindai Zhongxiyi Zhenglunshi). Hefei: Anhui Science and Technology Press, 1993.

36 Unschuld Paul U. Medicine in China: a history of ideas. Berkeley, CA: University of California Press, 1985.

37 Sivin N. Traditional medicine in contemporary China. Ann Arbor, MI: University of Michigan Press, 1987.

38 Rinpoche VR. Tibetan medicine: illustrated in original texts. Delhi: Indian Books Centre, 2001:3-4.

39 Cai J. A general history of Tibetan medicine (Zangyixue Tongshi). Xining: Qinghai People's Press, 2002:54-70.

40 Qiangbachilie. Tibetan medicine in China (Zhongguo de Zangyi). Beijing: Chinese Tibetan Study Press, 1996:1-18.

41 Ga Z. The mystic culture of Tibetan Buddhism: Tantrism (Zangchuan Fujiao Shenmi Wenhua-Mizong). Lhasa: Tibetan People's Press, 1996:156.

42 Bauer JL. Through the Tibetan looking glass. JAltern ComplementMed 2000;6:303-4.

43 Bodeker $\mathbf{G}$. Lessons on integration from the developing world's experience. BMJ 2001;322:164-7.

44 Hong CD. Complementary and alternative medicine in Korea: current status and future prospects. J Altern Complement Med 2001;7:S33-40.

45 Tibetan Medicine Administration. Tibetan medicine (In Chinese. ) Lhasa: Tibetan People's Press, 2003.

46 World Health Organization. Traditional medicine: growing needs and potential, 2002.http://whqlibdoc.who.int/hq/2002/WHO_EDM_2002.4.pdf (accessed 19 February 2006).

47 World Health Organization. Traditional medicine Fact sheet No 134. 2003 http://www.who.int/mediacentre/factsheets/fs134/en/ laccessed 19 February 2006). 